reality they are infinite, so that the method cannot be regarded as rigorous.

The concluding paragraph in Ellis's paper is,- "The method of this paper leads to some elegant results when applied to the definite integral $\int_{0}^{\infty} \theta^{-a x} d x$, but it is enough to point out this application, which involves no difficalty whatever." I do not see what application is here referred to, unless integrals of the form $\int_{0}^{\infty} \frac{e^{-a x^{*}}}{x^{n}} d x$ or $\int_{0}^{\infty} \frac{\Sigma A e^{-a x}}{x^{n}} d x$ are meant; bat the former is infinite for $n=1$, and the discussion of the latter is of little value, as $\Sigma \mathrm{A} e^{-a s}$, subject to the required conditions for the integral to be finite, includes no functions of any interest.

In connexion with Ellis's paper I may refer to one by Bronwin in vol. xxiv., p. 491, of the Philosophical Magazine (1844), in which several integrals are evaluated, to which Ellis's results, and those given in this communication, are at once applicable.

\title{
Note on Normals and the Surface of Centres of an Algebraical
} Surface. By Samdel RoBerts, M.A.

[Read March 13th, 1873.]

I.

There are several methods by which we can determine, in ordinary cases, the number of normals to a surface which can be drawn from an arbitrary point. We may, for instance, consider a system of concentric spheres, and find the number of these which touch the giren surface.

It must be assumed, however, that this has no special relation to the plane at infinity, or the imaginary circle there.

Let the order of the surface, its class, and the degree of the tangent cone be $m, n, a$. Then, since one sphere of the system can be drawn through any point $(\mu=1)$, one sphere touches any plane $(\nu=1)$, and one sphere touches auy line $(\rho=1)$, we have, by a formula of $D_{\theta}$ Jonquières $\mu n+v m+\rho a=n+m+a$.

For a surface, order $m$, without singularities,

$$
n=m(m-1)^{2}, \quad a=m(m-1), \quad m+n+a=m^{3}-m^{2}+m .
$$

2. If, however, the surface is specially related to the plane at infinity, the above formula fails. We will now endeavour to determine the number sought by finding how many normals can be drawn to the surface from a point in the plane at infinity. In general, the normals to a surface at points in the plane at infinity lie altogether in that plane. We have therefore altogether at infinity $m+a$ normals, and of course there are $n$ finite parallel normals, so that we get, as before,

$$
m+n+a
$$


Suppose now, as a first speciality, that the surface has in the plane at infinity certain nodal and cuspidal curves, these not being specially related to the imaginary circle there, and that the surface has certain nodes also infinitely distant. The formula is not affected by such an arrangement of the singularities quà arrangement. For, having allowed for the effect of these singularities in estimating the class and therefore the number of finite normals, we must not allow for their effect again in estimating the number of normals at infinity. In other words, we must count as multiple normals the lines through singular points of the section at infinity. The formula therefore remains unchanged.

3. Let us now suppose that the surface has $p$ contacts with the plane at infinity. We can still draw from any point $m+n+a$ normals or quasi normals. The quasi normals are $p$ lines directed towards the points of contact with the plane at infinity. Every finite line is analytically. normal to the plane at infinity, and therefore all lines directed towards the supposed points of contact are normal to the surface; but we must evidently exclude these as improper normals. Hence the formula now is

$$
m+n+a-p \text {. }
$$

It will be observed that here we shall have $p$ additional double points on the section at infinity. But if we reduce the number of the finite normals by $p$, it is necessary to reckon as double normals at infinity lines through these nodes.

4. If the section at infinity has a double contact with the imaginary circle, the section is of a circular nature. Any right line meets the circle normally, and therefore any right line through a point of contact with the circle is normal to the section. But these normals have to be rejected. Hence, for $2 q$ such separate contacts, the formula is

$$
m+n+a-2 q \text {. }
$$

When, however, these points of contact become singular points, as when the surface is one of revolution, we must proceed by finding the number of finite normals similarly directed, namely $n$, if $n$ is the class of an axial section. The section at infinity, consisting of say $m$ quasi circles for a surface of order $2 m$, gives us $2 m$ normals at infinity, and the number of normals is $n+2 m$. The surface may, however, be of odd order, $2 m+1$, we then have at infinity only $m$ quasi circles, but there is a line to which we can draw one normal, so that we get for the number of normals $n+2 m+1$.

5. We have next to consider the case in which the surface contains the imaginary circle. If a surface contains the circle in the order $r$, this 
in itself implies $2(m-2 r)$ singular points on the section, which may arise partly in one way, as from a multiple curve, and partly in another, as by points of contact at infinity. It hecomes necessary to regard the specialities of each case in this respect. The formula is of the form

$$
n+\mathrm{P} \text {, }
$$

where $P$ depends on the nature of the singularities of the non-circular part of the section, and $n$ is the number of finite normals.

Thus, in the case of a quartic surface on which the circle is nodul, the number of normals through a point is $n$, the class of the surface. When a cubic surface passes through the circle, so that the plane at infinity contains a line of the surface, this plane is doubly tangential, and at infinity we can draw one normal to the line. Hence the number of normals through a point is

$$
n-2+1=n-1 \text {. }
$$

For Dupin's parabolic cyclide this number is 3 , and for his general cyclide the number is 4 .

II.

6. The order and class of the surface of centres of a surface of the order $m$ without singular points and curves have been determined by M. Darboux (Comptes Rendus, 1870), and M. Löthar Marcks (Math. Annal., Clebsch and Neumann, B. v. H. 1). The order in this case is $2 m(m-1)(2 m-1)$, and the class $2\left(m^{3}-m^{2}-m\right)$.

I have sought to determine the order and class in the more general case where the surface possesses ordinary singularities, as nodal and cuspidal curves, nodes, \&c. The assumption is made, however, that the primitive surface is not specially related to the plane at infinity, i.e., that it does not touch that plane or pass through the imaginary circle, \&c. It is evident that such singularities materially affect the numbers songht, ws in the analogous cases of plane space.

In the first place, consider the series of normals to the surface parallel to a fixed plane. These normals will be those which can be diawn to the surface along the curve of contact of an enveloping cylinder whose axis is perpendicular to the fixed plane. The projections, therefore, of the normals in question in that plane form the series of normals to the section of the cylinder by the plane. We may say then that, to an eye placed at a very great distance, the axis of the cylinder being the axis of vision, the normals apparently envelope the evolute of the plane section.

- The apparently successive normals, drawn as mentioned, do not in general intersect. The apparent envelope is due to apparent intersections.

But it is important to remark that the points at infiuity of the evo- 
lute correspond to actual intersections of normals, and morcover of successive normals, and are therefore on the surface of centres. To show. this, consider first of all the normals at finite points of the curve of contact, whose projections intersect successively at infinitely distant points. Those normals must be coincident, or rather approximate, along their whole leugth, for normals with an apparent interval cannot be regarded as successive; and the projections are normal to the plane section of the cylinder at points of inflection which, as we know, correspond to parabolic points on the curre of contact. Hence the points at infinity now in question are the infinitely distant extremities of binormals of the curve of contact, which, as their name indicates, are normal to two successive elements of the curve, being perpendicular to the osculating planes. The points are therefore due to parabolic points, one of the principal radii of curvature being infinite at these points. They are simple points on the line at infinity determincd as the intersection with the plane at infinity of the fixed plane. Their number is equal to the number of inflections on tho plane section of tho cylinder.

7. Besides the points thus determined ou the line at infinity determined by the fixed plane, we have to consider the cusps of the evolute, which count each three times on that line which is their common tangent. These cusps are due to the points at infinily of the plane scction of tho cylinder, and these correspond to the points at infinity of the curve of contact.

The normals at these points are, by the doctrines of planc geometry, cuspidal tangents of the evolute, and their correspondents must in liko manner be successive twico over with regard to the surface.

Lot us consider the generators of the cylinder lying at infinity. Theso will be tangents to the corresponding section of tho surface at the points where the curve of contact mects the section, and pass through the infnitely distant points of the plane section of the cylinder. The cusps spoken of are harmonic conjugates to those points with regard to tho pair of circular points lying on the same line at infinity. In other words, the cusps are on the polar reciprocal of the section of the surface at infinity taken witl regard to the imnginary circle.

We may infer, then, by shifting our fixed plawe, that the locus of thoso cusps is the reciprocal of the section at infinity of tho surface with rospect to the imaginary circle, and that it counts thrce timos on tho corresponding section of the surface of centres, and is, in fuct, a cuspidal curve thereof, the plane at infinity being tangential all along it.

8. Further, however, the points on the line at infinity with which wn are dealing, which correspond to tho suction of the surface at infiuity, must be reckoned. The normals to the surfice along this section lio vol. IV.-NO. 60 . 
altogether at infinity, and envelope a quasi-evolute. The number of the points in question is therefore the order of this evolute.

9. Writing then-

Order of plane section of the primitive... $\quad \ldots, m$

$\begin{array}{llllllll}\text { Class } & \ldots & \ldots & \ldots & \ldots & \ldots & \ldots & a\end{array}$

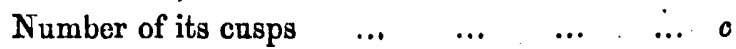

$\begin{array}{llllll} & \text { of its inflexions... } & \ldots & \ldots & \ldots & i\end{array}$

$\begin{array}{llllll}\text { Class of tangent cone } & \ldots & \ldots & \ldots & \ldots & n\end{array}$

Number of its cuspidal edges $\ldots . . \quad \ldots \quad \ldots k$

" of its stationary planes . ... ...

we have for the order of the surface of centres

$$
3 n+k(=3 a+\imath)+3 a+c(=3 m+i),
$$

a form bearing a remarkable analogy to the corresponding one for plane space.

10. To determine the class of the surface of centres, I make use of a theorem due, I believe, to M. Klein, and employed by the before-named authors.

If a ray system is of the order $m^{\prime}$ and class $n^{\prime}, i . e ., m^{\prime}$ lines meet in any point and $u^{\prime}$ lines lie in any plane, the difference of the order and class of the focal surface is

$$
\text { order-class }=2\left(m^{\prime}-n^{\prime}\right) \text {. }
$$

For such a system consisting of normals to a surface, the focal surface is the surface of centres, and

$$
m^{\prime}=m+n+a, \quad n^{\prime}=a, \quad \text { and therefore } m^{\prime}-n^{\prime}=n+n .
$$

As regards the formula $n^{\prime}=a$, observe that the points belonging to the normals which lie in a given plane are the intersections of the given plane by the curve of contact with the surface of the cylinder enveloped by the tangent planes which cut at right angles the given plane, i.e., which pass through the point at infinity on any perpendicular to the given plane; the number of normals is thus equal to tho order of the curve of contact, viz., it is $=a$.

We get, then, for the required number

$$
\begin{aligned}
3(n+a)+c & +\kappa-2(m+n) \\
& =n+3 a+c+\kappa-2 m \\
& =6 a+c+\imath-2 m-2 n,
\end{aligned}
$$

which for a surface of order $m$ without singularities is

$$
=2\left(m^{3}-m^{2}-m\right) \text {, }
$$

agreeing with Darboux.

11. Using accented letters for the corresponding characteristics of 
the reciprocal surface taken with regard to a centre of reciprocation not specially related to the surface, we may write

$$
\begin{aligned}
& c-k^{\prime}=3(m-a) \\
& c^{\prime}-k=3(n-a) ;
\end{aligned}
$$

whence

$$
\begin{aligned}
3(n+a)+c+k & =3(m+a)+c^{\prime}+\kappa, \\
n+3 a+c+\kappa-2 m & =m+3 a+c^{\prime}+\kappa^{\prime}-2 n ;
\end{aligned}
$$

that is, the order and class of the surface of centres of a surface and of that of its general reciprocal are the same.

What we require, then, to enable us to determine the order and class. of the surface of centres of a surface possessing ordinary singularities as a nodal and a cuspidal curve, nodes or singularities which can be estimated in terms of nodes, but not specially related to the plane at infinity, is to know

$$
\begin{array}{lccccccc}
\text { Order } & \ldots & \ldots & \ldots & \ldots & \ldots & \ldots & m \\
\text { Class } & \ldots & \ldots & \ldots & \ldots & \ldots & \ldots & n \\
\text { Rank } & \ldots & \ldots & \ldots & \ldots & \ldots & . \ldots & a \\
\text { Order of cuspidal } & \text { curre. } & \ldots & \ldots & \ldots & \ldots & 0 \\
\text { Class of spinode torse } & \ldots & \ldots & \ldots & \ldots & c^{\prime}
\end{array}
$$

The case of singularities at infinity, and especially in connection with the imaginary circle, requires separate discussion.

\section{April 10th, 1873.}

Dr. HIRST, F.R.S., President, in the Chair.

Mr. W. D. Niven, M.A., Fellow of Trinity College, Cambridge, was proposed for election; and Prof. Wolstenholme was admitted into the Society.

Prof. Clifford made a few remarks in correction of a statement be had made at the previous meeting, during the discussion on Mr, Hayward's paper.

Mr. J. W. L. Glaisher then proceeded to read his paper "On the calculation of the value of the Theoretical Unit-Angle to a great number of decimal places."

The following papers were discussed (in the absence of the authors) by Messrs. T. Cotterill, Clifford, Merrifield, and the President:- 
"On Systems of Porismatio Equations, Algebraical and Trigonometrical ;" "Note on Epicycloids and Hypocycloids ;" "Locus of Point of Concourse of Perpendicular Tangents to a Cardioid ;" and "Elliptic Motion under Acceleration constant in direction:" Prof. Wolstenholmo.

"On the Theory of a System of Electrified Conductors;" "On the Focal Lines of a Refracted Pencil :" Prof. J. Clerk Maxwell."

The following presents wero received :-

"Sur les trajectoires des points d'une droite mobile dans l'cspace," and "Démonstration géométrique d'une proposition duc à M. Bornard," by M. Mannheim : from the Author.

On the Oalculation of the Value of the Theoretical Unit.Angle to a Great Number of Docimal.Places. By J. W. L. Graisher, B.A., Fellow of Trinity College; . Cambridge.

$$
\text { [Read April 10th, 1873.] }
$$

The number of degrees in the theoretical unit of angular measure (viz., the angle the length of whose arc is equal to the radius) is $\frac{180}{\pi}$, so that merely by a division this angle can be determined to about as many decimals of a degree as there are decimals of $\pi$ known. More than a year ago $I$ had need of the value of the unit-angle to a much greater degree of accuracy than it was given to in any place that I was acquainted with, and to make certain of having an abundant number of decimals, I had the division performed, taking as the divisor the first fifty-four figures of $\pi$ (this being a good point to break off at, as the fifty-fifth figure is a cipher), and shortly afterwards $I$ had the same work performed by another computer, nearly independently. Having in the course of the year examined a great number of tables, collections of constants, \&c., and never having met with the angle in question given to even a moderate number of places, I thought it would be desirable to communicate the result of the calculation to tho Society. I havo accordingly examined one of the calculations myself, and have also had the division performicd a third time, so that the prescnt results are submitted with confidence.

The theoretical unit-angle to 52 decimals of a degree is

$\begin{array}{rrrrr}57^{\circ} \cdot 29577 & 95130 & 82320 & 87679 & 81548 \\ 14105 & 17033 & 24054 & 72466 & 56432 \\ 15 \ldots & & & & \end{array}$

